\title{
TRESK gene recombinant adenovirus vector inhibits capsaicin-mediated substance $P$ release from cultured rat dorsal root ganglion neurons
}

\author{
JUN ZHOU ${ }^{1}$, SHANG-LONG YAO ${ }^{2}$, CHENG-XIANG YANG ${ }^{1}$, \\ JI-YING ZHONG ${ }^{1}$, HAN-BING WANG ${ }^{1}$ and YAN ZHANG ${ }^{2}$ \\ ${ }^{1}$ Department of Anesthesiology, First People's Hospital of Foshan, Foshan, Guangdong; ${ }^{2}$ Department of Anesthesiology, \\ Union Hospital, Tongji Medical College, Huazhong University of Science and Technology, Wuhan, Hubei, P.R. China
}

Received October 27, 2011; Accepted January 19, 2012

DOI: $10.3892 / \mathrm{mmr} .2012 .778$

\begin{abstract}
The present study was conducted to determine whether the activation of TRESK in the dorsal root ganglion (DRG) by the TRESK gene recombinant adenovirus vector inhibits the capsaicin-evoked substance $\mathrm{P}(\mathrm{SP})$ release using a radioimmunoassay. TRESK is an outwardly rectifying $\mathrm{K}^{+}$ current channel that contributes to the resting potential and is the most important background potassium channel in DRG. Previous studies have shown that neuropathic pain (NP) is closely related to the regulation of certain potassium channels in DRG neurons, while DRG-released SP is important in the peripheral mechanism of NP. In the present study, the TRESK gene adenovirus vector significantly enhanced the TRESK mRNA and protein of the cultured rat DRG neurons. Radioimmunoassay analysis revealed that the capsaicin-mediated SP release was significantly inhibited by the TRESK gene recombinant adenovirus vector in rat DRG neurons. These findings suggest that TRESK plays a role in adjusting the release of SP in DRG, which is related to NP.
\end{abstract}

\section{Introduction}

TRESK, the most recently reported type of two-pore potassium ion channel, is expressed in the center and peripheral nervous system, including the cerebellum, the cerebral cortex and the dorsal root ganglia (DRG) (1). TRESK is an outwardly rectifying $\mathrm{K}^{+}$current channel that contributes to the resting potential and is the most important background potassium channel with a relatively small conductance. Neuropathic pain (NP) is closely related to the regulation of certain potassium channels in DRG neurons. It was previously shown that the

Correspondence to: Dr Jun Zhou, Department of Anesthesiology, First People's Hospital of Foshan, Foshan, Guangdong 528000, P.R. China

E-mail: zhoujun7843@126.com

Key words: dorsal root ganglion neuron, TRESK, subtance P release change of TRESK is likely to be involved in the development of NP (2-4).

Capsaicin is a selective sensory nerve stimulator, and small doses of it excite sensory nerve endings, releasing nerve transmitters $(5,6)$. Substance $\mathrm{P}(\mathrm{SP})$, as a nerve transmitter, is a member of the tachykinin family, which is widely distributed in the peripheral nervous system. SP functions mainly as a nociceptive stimulus receptor, and is also involved in inflammatory response $(7,8)$. Numerous studies have demonstrated that DRG-released SP is important in the peripheral mechanism of NP.

We recently examined the expression level of TRESK mRNA in the DRG of NP of rats. Results of that study demonstrated that downregulation of the TRESK background current in DRG may be related to NP (9). In addition, rat TRESK fulllength cDNA was cloned and recombinant TRESK adenovirus vector was constructed successfully in our previous study (10).

In the present study, therefore, we examined whether the activation of TRESK detected by TRESK mRNA and protein by the TRESK gene recombinant adenovirus vector inhibits the capsaicin-evoked SP release in DRG neurons.

\section{Materials and methods}

Materials. TRESK adenovirus vector (pAdTrack-CMVTRESK), negative adenovirus, PCR primer (Shanghai R\&S Biotechnology Co., Ltd., Shanghai, China); the rabbit polyclonal anti-TRESK antibody, TRESK-conjugated anti-rabbit antibody (Santa Cruz Biotechnology, Santa Cruz, CA, USA); the SP radio-immunity kit (the Second Army Medical College, Shanghai, China); the Superscript kit (Life Technologies, Gaithersburg, MD, USA); and the DNA engine Opticon 2 real-time PCR detection system (Bio-Rad, Hercules, CA, USA) were used in this study.

Isolation and culture of DRG neurons. DRGs were removed from young adult SD rats (6-9 weeks) and were dissociated into single isolated neurons by enzyme treatment of $0.125 \%$ collagenase for $90 \mathrm{~min}$ (twice), followed by $0.25 \%$ trypsin for $30 \mathrm{~min}$ at $37^{\circ} \mathrm{C}$ and by trituration with fire-polished Pasteur pipettes of decreasing tipdiameter (11). The cells 
(2-3 DRG/well) were subsequently plated on polyethyleneimine and laminin-coated 96-well tissue-culture plates and incubated in DMEM containing 10\% heat-inactivated horse serum, $1 \%$ penicillin/streptomycin and $200 \mathrm{mM}$ glutamine. The cultures were maintained at $37^{\circ} \mathrm{C}$ in a water-saturated atmosphere with $5 \% \mathrm{CO}_{2}$ for 5 days prior to the experiment. On the fifth day of culture, neurons exhibited globular cell bodies and extended axonal processes. Various non-neuronal cells, such as Schwann cells, fibroblasts and satellite cells, were also present as an oxford gray cellular background. Approval was received by the First People's Hospital of Foshan ethics committee.

Experimental groups. DRG neurons were cultured in 54 wells and divided randomly into six groups, 9 holes per group, per well. Group $\mathrm{C}$ received no treatment. Each well in the $\mathrm{S}$ group was incubated for $10 \mathrm{~min}$ with fresh $300 \mathrm{nmol} / \mathrm{l}$ capsaicin culture medium. Each well in the $\mathrm{NC}$ group was administered $3 \times 10^{7}$ negative adenovirus (Shanghai R\&S Biotechnology Co., Ltd.). Each well in the NCS group was given $3 \times 10^{7}$ negative adenovirus and then incubated for $10 \mathrm{~min}$ with fresh $300 \mathrm{nmol} / \mathrm{l}$ capsaicin culture medium $72 \mathrm{~h}$ later. Each well in the R group was given $3 \times 10^{7}$ pAdTrack-CMV-TRESK (Shanghai R\&S Biotechnology Co., Ltd.). Each well in the RS group was given $3 \times 10^{7}$ pAdTrack-CMV-TRESK and then incubated for $10 \mathrm{~min}$ with fresh $300 \mathrm{nmol} / \mathrm{l}$ capsaicin culture medium $72 \mathrm{~h}$ later.

Measurement of TRESK $m R N A$. Total RNA harvested from 3 wells of each group by the acid guanidinium thiocyanate-phenolchloroform extraction method was separately subjected to reverse transcription into cDNA using a Superscript kit (Life Technologies), according to the manufacturer's protocol. cDNA sample $(2 \mu \mathrm{g})$ was used immediately in a real-time PCR assessment of TRESK mRNA levels with iQ SYBR-Green Supermix, and forward primer (5'-GGTGCCAACGATGATCT-3') and reverse primer (5'-CTGCTGGGCTGTGGGTCTAG-3') on a DNA engine Opticon 2 real-time PCR detection system (Bio-Rad). The thermal cycler parameters used were: denaturation of 1 cycle of $2 \mathrm{~min}$ at $95^{\circ} \mathrm{C}$, followed by 40 cycles of $20 \mathrm{sec}$ at $95^{\circ} \mathrm{C}$, annealing for $15 \mathrm{sec}$ at $57^{\circ} \mathrm{C}$ and extension $20 \mathrm{sec}$ at $72^{\circ} \mathrm{C}$. A glyceraldehyde-3-phosphate dehydrogenase (GAPDH) control was run simultaneously with the same reaction recipe listed in the instructions manual for the iQ SYBR-Green Supermix. The $2^{-\Delta \Delta C t}$ method was used to calculate the expression level of TRESK mRNA (12).

Measurement of TRESK protein. Three wells were removed from each group, and the cells were processed using western blotting. Primary antibodies were raised against TRESK (1:1,000 dilution; rabbit polyclonal TRESK antibodies; Santa Cruz) or $\beta$-actin. After washing, the membranes were further incubated with horseradish peroxidase-conjugated anti-rabbit secondary antibody (1:2,000 dilution; Santa Cruz Biotechnology) for $1 \mathrm{~h}$ at room temperature $\left(22^{\circ} \mathrm{C}\right)$. The proteins were then used for the detection of chemiluminescence, according to the manufacturer's instructions.

Measurement of SP content. We measured the SP content of the culture medium (serum-free DMEM) in the last 3 wells from each group at $72 \mathrm{~h}$ following grouping. Cells were removed from the medium and were maintained for 10 min with $300 \mathrm{nmol} / 1$ capsaicin in groups S, NCS and RS. Subsequently, the medium from the cells in each group was collected and centrifuged at 1,200 x g for $5 \mathrm{~min}$, followed by a radioimmunoassay with the SP radioimmunity kit (the Second Army Medical College, Shanghai, China) of the medium (13).

Statistical analysis. The data are presented as the means \pm SD. Statistical analyses were performed by the multiple t-test with the Bonferroni correction following ANOVA. P<0.05 was considered to indicate a statistically significant difference.

\section{Results}

Comparison of TRESK mRNA from cultured DRG neurons of each group. In our previous studies, we successfully constructed the recombinant TRESK adenovirus vector (pAdTrack-CMV-TRESK). This vector was found to upregulate TRESK mRNA expression levels in the DRG neurons of rats (10).

Therefore, we investigated whether these mass duplicate pAdTrack-CMV-TRESK are affected in the enhancement of TRESK mRNA from cultured DRG neurons exposed to long-term (72 h) treatment with adenovirus vector. Fig. 1A shows that the TRESK mRNA from cultured DRG neurons was significantly enhanced when exposed to long-term (72 h) treatment with pAdTrack-CMV-TRESK in groups $\mathrm{R}$ and RS. However, no increase in the TRESK mRNA levels of the rat DRG neurons was observed following a 72-h exposure of negative adenovirus in groups $\mathrm{NC}$ and NCS, as was the case with groups $\mathrm{C}$ and $\mathrm{S}$ as controls (data not shown).

Comparison of TRESK protein from cultured DRG neurons of each group. The protein levels of TRESK exposed to long-term (72 h) treatment with pAdTrack-CMV-TRESK or negative adenovirus vector were examined using western blotting. The increase in the protein levels of TRESK from cultured DRG induced by pAdTrack-CMV-TRESK was significantly enhanced when exposed to long-term (72 h) treatment in groups R and RS. However, no increase in the TRESK protein levels of the rat DRG neurons was observed following a 72-h exposure of negative adenovirus in groups NC, NCS, C and S as controls (Fig. 1B) (data not shown).

Comparison of capsaicin-induced SP release from cultured $D R G$ neurons of each group. To investigate whether pretreatment with upregulation of TRESK mRNA causes the attenuation of SP release evoked by capsaicin, the levels of capsaicin-induced SP release from cultured DRG neurons pretreated with pAdTrack-CMV-TRESK or negative adenovirus vector were examined. Fig. 1C shows that the release of SP from cultured DRG neurons was significantly enhanced when incubated for 10 min with capsaicin in groups S, NCS and RS. Although the capsaicin-evoked SP release was significantly enhanced by pre-treatment with pAdTrack-CMV-TRESK in comparison to the control group which was not incubated with capsaicin, the capsaicin-evoked SP release from cultured DRG was significantly attenuated by exposure to long-term (72 h) treatment with pAdTrack-CMV-TRESK in the RS group compared to the NCS and S groups. There was no significant 

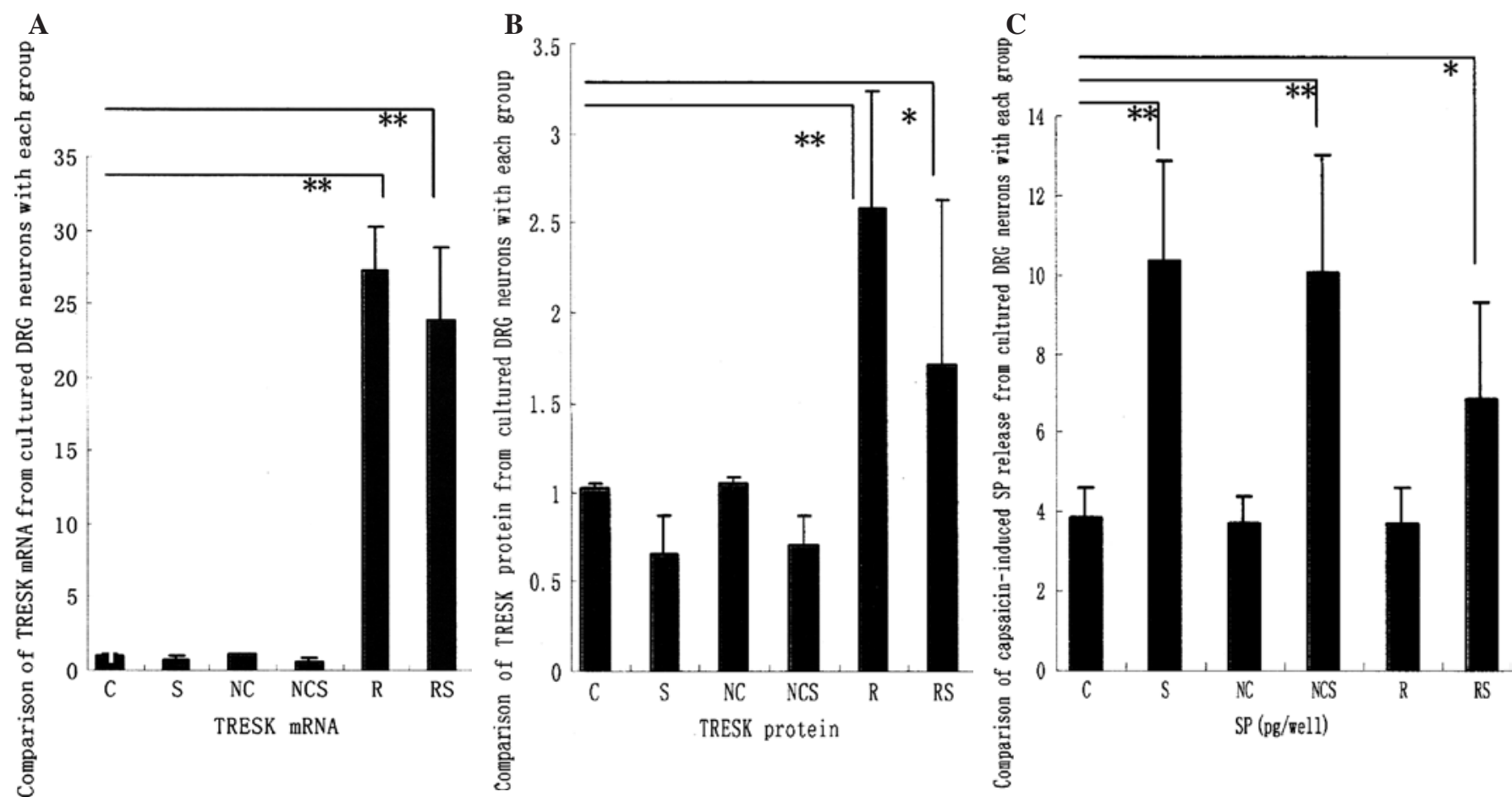

Figure 1. Comparison of TRESK mRNA, TRESK protein and capsaicin-induced SP release from the cultured DRG neurons of each group. (A) TRESK mRNA was detected by RT-PCR. (B) TRESK proteins were detected by western blotting. (C) Capsaicin-induced SP release from DRG neurons was assessed by RNA. Values are expressed as the means $\pm \mathrm{SD}$ of five independent experiments. ${ }^{*} \mathrm{P}<0.05 ;{ }^{* *} \mathrm{P}<0.01$.

difference in the release of SP from cultured DRG neurons in the R, NC and C groups (data not shown).

\section{Discussion}

Results of the present study show that long-term (72 h) exposure of cultured rat DRG neurons to the pAdTrack-CMV-TRESK resulted in the enhancement of TRESK mRNA, causing the protein levels of TRESK to increase, leading to the attenuation of SP release triggered by capsaicin.

Two-pore potassium is now considered an important ion channel involved in the pain mechanism $(14,15)$. As a subtype of two-pore potassium channel, TRESK is similar in distribution, physiology and pharmacology to other subtypes, which are related to pain $(1,3,4)$. TRESK was found to be greatly expressed in the DRG neurons of rats, and it was the main background current in DRG at 37 or $24^{\circ} \mathrm{C}(2,16)$. In particular, many studies have demonstrated that DRG is important in the peripheral mechanism of NP $(17,18)$. These findings show that TRESK may be involved in the development of NP. Furthermore, gliocyte is involved in the occurrence and maintainance of NP, while it exhibits permeability for potassium channel at resting potential. As a background potassium current for resting potential, TRESK is likely to have a key role in the excitation mechanism of gliocyte $(19,20)$.

SP is an 11-amino acid peptide sensory neurotransmitter, which is synthesized in the DRG and released from primary afferent neurons to convey information regarding various noxious stimuli.

SP release from primary afferent neurons is a highly complex process that often involves critical intracellular effectors, such as ion channel $(21,22)$. Further investigations are required to ascertain whether one or more substances are involved in the regulation of SP release by the application of capsaicin.

Previously, we cloned the rat TRESK full-length cDNA and successfully constructed the recombinant TRESK adenovirus vector. This vector effectively upregulated TRESK mRNA and protein levels in the DRG neurons of rat (10). Therefore, the present findings indicate that the mass duplicate pAdTrack-CMV-TRESK may be a means of clarifying whether any change in TRESK in DRG neurons is involved in the progression of NP. Our results demonstrated that the upregulation of TRESK in DRG neurons is involved in the regulation of capsaicin-evoked SP release. It is thus possible that TRESK is involved in the mechanism of NP.

Following our previous study (9), the present findings demonstrate that the modulation of TRESK of DRG neurons is involved in NP; consequently, another mechanism of NP is possible: TRESK has the function which maintains the balance of resting potential in the DRG neurons. When the DRG neurons accepted the signal from peripheroceptor, such as acid or other hurt stimulation, it immediatedly downregulates TRESK and then induces the polarization of neurons. Additionally, the increase of the introvertive potassium current evokes the release of SP and then delivers more signal transmitters to the central receptor $(23,24)$. If the hurt stimulation cannot be relieved, acute pain changes into NP.

By contrast, the release of SP is regulated with calcineurin. Moreover, a strong and persistent stimulation, such as peripheral nervous injury, clearly decreases the release of calcineurin (25-27). Therefore, the fact that calcineurin agonist cures chronic pain indicates that it maintains the TRESK current to regulate the release of SP to inhibit the development of NP. 
In conclusion, we have demonstrated that the activation of TRESK by its gene recombinant adenovirus vector induces the enhancement of TRESK mRNA in the DRG neurons of rats, thereby raising the protein levels of TRSK and attenuating the capsaicin-evoked SP release from cultured DRG neurons. It is possible that TRESK in DRG has an accommodating effect on the SP release process. These observations provide evidence that TRESK in DRG may be involved in the progression of NP through the neuromodulatory actions of SP.

\section{References}

1. Kang D, Mariash E and Kim D: Functional expression of TRESK-2, a new member of the tandem-pore $\mathrm{K}^{+}$channel family. J Biol Chem 279: 28063-28070, 2004.

2. Kang D and Kim D: TREK-2(K2P 10.1) and TRESK(K2P 18.1) are major background $\mathrm{K}^{+}$channels in dorsal root ganglion neurons. Am J Physiol Cell Physiol 291: 138-146, 2006.

3. Liu C, Au JD, Zou HL, Cotten JF and Yost CS: Potent activation of the human tandem pore domain K channel TRESK with clinical concentrations of volatile anesthetics. Anesth Analg 99: $1715-1722,2004$

4. Keshavaprasad B, Liu C, Au JD, Kindler CH, Cotten JF and Yost CS: Species-specific differences in response to anesthetics and other modulators by the K2P channel TRESK. Anesth Analg 101: 1042-1049, 2005.

5. Rigoni M, Trevisani M, Gazzieri D, et al: Neurogenic responses mediated by vanilloid receptor-1 (TRPV1) are blocked by the high affinity antagonist, iodo-resiniferatoxin. Br J Pharmacol 138: 977-985, 2003.

6. Nemeth J, Reglodi D, Pozsgai G, et al: Effect of pituitary adenylate cyclase activating polypeptide-38 on sensory neuropeptide release and neurogenic inflammation in rats and mice. Neuroscience 143: 223-230, 2006.

7. Carter MS and Krause JE: Structure, expression and some regulatory mechanisms of the rat preprotachykinin gene encoding substance $\mathrm{P}$, neurokinin $\mathrm{A}$, neuropeptide $\mathrm{K}$ and neuropeptide gamma. J Neurosci 10: 2203-2214, 1990.

8. Ribeiro-da-Silva A and Hökfelt T: Neuroanatomical localisation of substance P in the CNS and sensory neurons. Neuropeptides 34 : 256-271, 2000.

9. Zhou J, Yao SL, Yang CX, et al: Changes in expression of TRESK mRNA in dorsal root ganglion in a rat model of neuropathic pain Chin J Anesthesiol 31: 183-185, 2011.

10. Zhou J, Yao SL, Yang CX, et al: Construction of the recombinant adenovirus vector of rat TRESK gene. Chin J Anesthesiol 31: 296-298, 2011.

11. Tang HB, Inoue A, Oshita K and Nakata Y: Sensitization of vanilloid receptor 1 induced by bradykinin via the activation of second messenger signaling cascades in rat primary afferent neurons. Eur J Pharmacol 498: 37-43, 2004.
12. Halfon $\mathrm{P}$, Bourlière $\mathrm{M}$, Pénaranda $\mathrm{G}$, Khiri H and Ouzan D: Real-time PCR assays for hepatitis C virus (HCV) RNA quantitation are adequate for clinical management of patients with chronic HCV infection. J Clin Microbiol 44: 2507-2511, 2006.

13. Inoue A, Hashimoto $\mathrm{T}$, Hide I, et al: 5-Hydroxytrytaminefacilitated release of substance $\mathrm{P}$ from rat spinal cord slices is mediated by nitric oxide and cyclic GMP. J Neurochem 68: 128-133, 1997.

14. Viana F, de la Peña E and Belmonte C: Specificity of cold thermotransduction is determined by differential ionic channel expression. Nat Neurosci 5: 254-260, 2002.

15. Alloui A, Zimmermann K, Mamet J, et al: TREK-1, a $\mathrm{K}^{+}$channel involved in polymodal pain perception. EMBO J 25: 2368-2376, 2006.

16. Dobler T, Springauf A, Tovornik S, et al: TRESK two-poredomain $\mathrm{K}^{+}$channels constitute a significant component of background potassium currents in murine dorsal root ganglion neurones. J Physiol 585: 867-879, 2007.

17. Baumann TK, Burchiel KJ, Ingram SL and Martenson ME: Responses of adult human dorsal root ganglion neurons in culture to capsaicin and low pH. Pain 65: 31-38, 1996.

18. Baumann TK, Chaudhary P and Martenson ME: Background potassium channel block and TRPV1 activation contribute to proton depolarization of sensory neurons from humans with neuropathic pain. Eur J Neurosci 19: 1343-1351, 2004.

19. Wen YR, Suter MR, Kawasaki Y, et al: Nerve conduction blockade in the sciatic nerve prevents but does not reverse the activation of p38 mitogen-activated protein kinase in spinal microglia in the rat spared nerve injury model. Anesthesiology 107: 312-321, 2007.

20. Guo W, Wang H, Watanabe M, et al: Glial-cytokine-neuronal interactions underlying the mechanisms of persistent pain. J Neurosci 27: 6006-6018, 2007.

21. Tominaga M, Numazaki M, Iida T, et al: Regulation mechanisms of vanilloid receptors. Novartis Found Symp 261: 4-12, 2004

22. NakagawaH and Hiura A: Capsaicin, transient receptor potential (TRP) protein subfamilies and the particular relationship between capsaicin receptors and small primary sensory neurons. Anat Sci Int 81: 135-155, 2006.

23. Smith HS: Arachidonic acid pathways in nociception. J Support Oncol 4: 277-287, 2006.

24. Woo YC, Park SS, Subieta AR and Brennan TJ: Changes in tissue, $\mathrm{Ph}$ and temperature after incision indicate acidosis may contribute to postoperative pain. Anesthesiology 101: 468-475, 2004.

25. Czirják G and Enyedi P: Targeting of calcineurin to an NFAT-like docking site is required for the calcium-dependent activation of the background $\mathrm{K}^{+}$channel, TRESK. J Biol Chem 281: 14677-14682, 2006.

26. Czirják G and Enyedi P: Zinc and mercury ions distinguish TRESK from the other two-pore-domain $\mathrm{K}^{+}$channels. Mol Pharmacol 69: 1024-1032, 2006.

27. Czirják G, Tóth ZE and Enyedi P: The two-pore domain $\mathrm{K}^{+}$ channel, TRESK, is activated by the cytoplasmic calcium signal through calcineurin. J Biol Chem 279: 18550-18558, 2004. 